\title{
Exosomes derived from miR-122-modified adipose tissue-derived MSCs increase chemosensitivity of hepatocellular carcinoma
}

\author{
Guohua Lou ${ }^{1}$, Xiuli Song ${ }^{2}$, Fan Yang ${ }^{1}$, Shanshan Wu' ${ }^{1}$ Jing Wang ${ }^{1}$, Zhi Chen ${ }^{1 *}$ and Yanning Liu ${ }^{\text {** }}$
}

\begin{abstract}
Background: Hepatocellular carcinoma (HCC) displays high resistance to conventional chemotherapy. Considering that microRNA-122 (miR-122) performs an essential function to promote chemosensitivity of HCC cells, an effective vehicle-mediated miR-122 delivery may represent a promising strategy for HCC chemotherapy. An increasing interest is focused on the use of exosomes as biological vehicles for microRNAs (miRNA) transfer. Mesenchymal stem cells (MSCs) are known for their capacity to produce large amounts of exosomes. This study aimed to determine whether adipose tissue-derived MSC (AMSC) exosomes can be used for miR-122 delivery.

Methods: AMSCs were transfected with a miR-122 expression plasmid. At $48 \mathrm{~h}$ after transfection, AMSC-derived exosomes (122-Exo) were harvested and added to recipient HCC cells. Expression levels of miR-122 in AMSCs, exosomes, and HCC cells were quantified by real-time PCR. The mRNA and protein levels of miR-122-target genes in recipient HCC cells were quantified by real-time PCR and Western blot, respectively. The effects of 122-Exo on cell viability, apoptosis, and cell cycle of HCC cells were evaluated by MTT and flow cytometry analysis. Xenograft models were used to determine whether 122-Exo can sensitize HCC cells to sorafenib in vivo.
\end{abstract}

Results: Data showed that miR-122-transfected AMSC can effectively package miR-122 into secreted exosomes, which can mediate miR-122 communication between AMSCs and HCC cells, thereby rendering cancer cells sensitive to chemotherapeutic agents through alteration of miR-122-target gene expression in HCC cells. Moreover, intra-tumor injection of 122-Exo significantly increased the antitumor efficacy of sorafenib on HCC in vivo.

Conclusions: The findings suggest that the export of miR-122 via AMSC exosomes represents a novel strategy to enhance HCC chemosensitivity.

Keywords: Adipose tissue-derived MSC, Exosome, miR-122, Hepatocellular carcinoma, Chemosensitivity

\section{Background}

Most hepatocellular carcinoma (HCC) patients are diagnosed at intermediate advanced stages, during which the only proven therapies are transarterial chemoembolization (TACE) and targeted therapy with the multikinase inhibitor, sorafenib [1]. However, HCC displays high resistance to commonly used chemotherapeutic agents, such as 5fluorouracil (5-FU) and doxorubicin. Therefore, the

\footnotetext{
* Correspondence: zju.zhichen@gmail.com; rainyliu2002@163.com 'State Key Laboratory for Diagnosis and Treatment of Infectious Diseases, The First Affiliated Hospital, College of Medicine, Zhejiang University, Collaborative Innovation Center for Diagnosis and Treatment of Infectious Diseases, 79\# Qingchun Road, 6A-17, Hangzhou 310003, China Full list of author information is available at the end of the article
}

discovery of new targets and the development of novel therapeutic approaches to enhance HCC chemosensitivity are urgently needed.

At present, certain progress is being developed in the abovementioned field $[2,3]$. Several studies have indicated that non-coding RNA, such as long non-coding RNAs and microRNAs (miRNAs), participate in cancer development and perform important functions in diagnosis and prognosis [4-6]. Moreover, miRNAs are determined to be correlated with chemosensitivity in cancers $[7,8]$. The liver-specific microRNA-122 (miR-122) has been found to perform multiple functions in liver physiology and pathology [9]. Notably, the loss or downregulation of miR-122 has been associated with HCC development and

\section{Biomed Central}

(C) 2015 Lou et al. Open Access This article is distributed under the terms of the Creative Commons Attribution 4.0 International License (http://creativecommons.org/licenses/by/4.0/, which permits unrestricted use, distribution, and reproduction in any medium, provided you give appropriate credit to the original author(s) and the source, provide a link to the Creative Commons license, and indicate if changes were made. The Creative Commons Public Domain Dedication waiver (http://creativecommons.org/publicdomain/zero/1.0/) applies to the data made available in this article, unless otherwise stated. 
progression [10] and is closely related to poor prognosis and metastasis of HCC [11]. Increasing evidence indicates that miR-122 can modulate the chemosensitivity of HCC cells [12]. Ectopic expression of miR-122 in nonexpressing HepG2 and Hep3B cells can inhibit tumorigenic properties, such as growth, invasion, and tumor formation in nude mice, as well as can sensitize these cells to doxorubicin and sorafenib $[13,14]$.

However, a safe and effective vehicle for miR-122 delivery also is a key factor in miR-122-mediated chemotherapy sensitization. Growing interest is focused on the use of exosomes as biological delivery vehicles for miRNA transfer, as exosomes do not elicit acute immune rejection and risk tumor formation [15]. Furthermore, exosomes can be manufactured in culture by incorporating therapeutic miRNAs into exosomeproducing cells, thereby possibly enabling personalized treatment [16]. Among the cell types known to produce exosomes, human mesenchymal stem cells (MSCs) are the most prolific producers [17]. Infusion of human MSC-derived exosomes into an immunocompetent mouse model of acute myocardial ischemia has been shown to be therapeutically effective and lacking of evident adverse effects [18].

Adipose tissue-derived MSCs (AMSCs) represent a highly advantageous tool for stem cell-based therapy [19]. The current study investigated whether exosomemediated transfer of miR-122 via miRNA-modified AMSCs can enhance the chemosensitivity of HCC cells.

\section{Results}

\section{AMSCs package miR-122 into secreted exosomes}

AMSCs positively expressed CD29, CD44, CD73, CD90, and CD105 but negatively expressed CD31, CD34, CD45, and HLA-DR. Positive staining of Oil Red O or Alcian Blue was observed after adipogenic induction of AMSCs for 14 days or chondrogenic induction for 28 days, respectively (Fig. 1).

AMSCs were transfected with a plasmid encoding for miR-122 or for cel-miR-67 (Caenorhabditis elegans miR67) as control. At $48 \mathrm{~h}$ after transfection, extracellular exosomes were isolated from the AMSCs supernatant. AMSC-derived exosomes showed the positive expression of exosomal markers, such as CD9, CD63, and CD81 [17, 20] (Fig. 2a). Afterward, miR-122 expression was measured in AMSCs and exosomes. The expression of miR-122 was $39.7 \pm 1.3$-fold and $21.6 \pm 3.4$-fold higher in miR-122-transfected AMSCs (AMSC-122) and their exosomes (122-Exo) than that of miR-122 in cel-miR67-transfected AMSCs (AMSC-67) and their exosomes (67-Exo), correspondingly (Fig. 2b, c). These data demonstrate that AMSCs can efficiently package plasmidexpressed miR-122 into secreted exosomes.

\section{Exosomes mediate miR-122 communication between AMSCs and HCC cells}

AMSC-122 cells were labeled with a phospholipid membrane dye, $\mathrm{DilC}_{16}$ (3), to trace the derived exosomes. After culturing for an additional $48 \mathrm{~h}$, fluorescent exosomes were collected and added to recipient HepG2 cells. Confocal imaging revealed the delivery of labeled exosomes as indicated by the presence of the fluorescent membrane in unlabeled recipient HepG2 cells (Fig. 2e, f). As a further proof, the expression of miR-122 was $10.5 \pm 1.4$-fold higher in 122-Exo-treated HepG2 cells than that in 67-Exo-treated cells (Fig. 2d). Thus, AMSC-derived exosomes can deliver miR-122 into HCC cells in vitro.

\section{2-Exo alters target gene expression in HCC cells}

To examine whether 122-Exo mediated-miR-122 communication can alter the expression of miR-122 target genes, such as cyclin G1 (CCNG1), a disintegrin and metalloprotease 10 (ADAM10), and insulin-like growth factor receptor 1 (IGF1R) in hepatoma cells [13, 14], HepG2 cells were exposed to 122-Exo or 67-Exo for $24 \mathrm{~h}$. Both mRNA and protein levels of these genes were downregulated in 122-Exo-treated HepG2 cells in comparison with 67-Exo-treated cells (Fig. 3).

These data suggest that miR-122, which is delivered via AMSC exosomes, is functionally active in acceptor HCC cells. Moreover, 122-Exo may potentially facilitate the sensitivity of HCC cells to chemotherapeutic agents by negative regulation of the expression of miR-122 target genes, which are involved in the drug resistance or sensitivity of cancer cells.

\section{2-Exo increases chemosensitivity of HCC cells}

To determine whether 122-Exo affects the chemosensitivity of hepatoma cells in vitro, HepG2 and Huh7 cells were exposed to chemotherapeutic agents combined with 122-Exo or 67-Exo. The growth inhibition of 5-FU or sorafenib on HCC cells was not altered by 67-Exo treatment, whereas the inhibitory effect of 5-FU or sorafenib on 122-Exo-treated HCC cells significantly increased in comparison with 67-Exo-treated control, particularly on HepG2 cells (Fig. 4a).

Flow cytometric analysis with Annexin V/PI staining also revealed an increase of apoptotic cells among 122Exo-treated HCC cells, especially in HepG2 cells, relative to 67-Exo-treated cells (Fig. 4b, c). The apoptotic rate between 67-Exo-treated and chemotherapeutic agent only-treated HCC cells presents no difference.

Subsequently, we tested whether cell cycle arrest contributed to the enhanced growth inhibition by 122-Exo treatment. As shown in Fig. 4e, treatment of HepG2 cells with 122-Exo resulted in an increase in the percentage of G0/G1 population from $67.0 \pm 1.6 \%$ (5-FU) and 

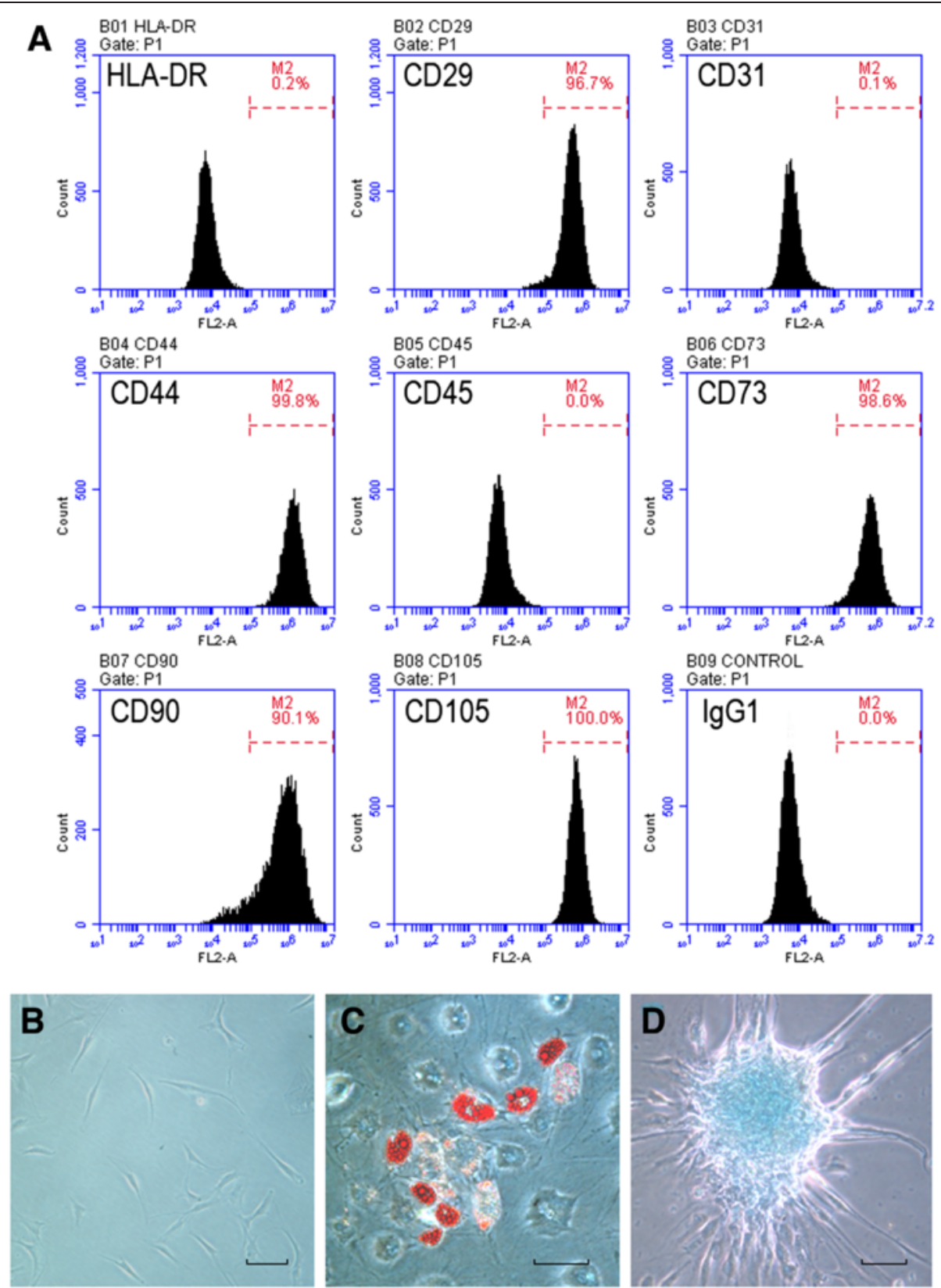

Fig. 1 Identification of human adipose tissue-derived mesenchymal stem cells (AMSCs). a Flow cytometry analysis of the surface markers in AMSCs. b Cellular morphology of AMSCs in culture. c Oil Red O staining in AMSCs cultured in adipogenesis differentiation medium for 14 days. d Alcian Blue staining in AMSCs cultured in chondrogenesis differentiation medium for 21 days. Scale bar $=50 \mu \mathrm{m}$

$63.4 \pm 1.1 \%$ (sorafenib) to $80.4 \pm 1.7 \%$ (122-Exo and 5FU) and $74.7 \pm 3.1 \%$ (122-Exo and sorafenib) with 5-FU or sorafenib exposure, respectively. Moreover, the treatment of Huh7 cells with 122-Exo showed similar G0/G1 arrest trends.

These data indicate that exosomes from miR-122modified AMSCs can increase the chemosensitivity of HCC cells by enhancing cell apoptosis and cell cycle arrest.

\section{2-Exo sensitizes HCC cells to sorafenib in vivo}

Finally, to determine whether 122-Exo could sensitize hepatoma cells to chemotherapeutic agents in vivo, AMSCderived exosomes (50 $\mu \mathrm{g}$ total protein in $10 \mu \mathrm{l}$ volume) were administered to nude mice bearing HepG2 cells combined with sorafenib treatment. One intra-tumor injection of 122-Exo at 7 days after subcutaneous inoculation significantly reduced the tumor volume and weight compared with 67-Exo or vehicle-treated control. The tumor volume 

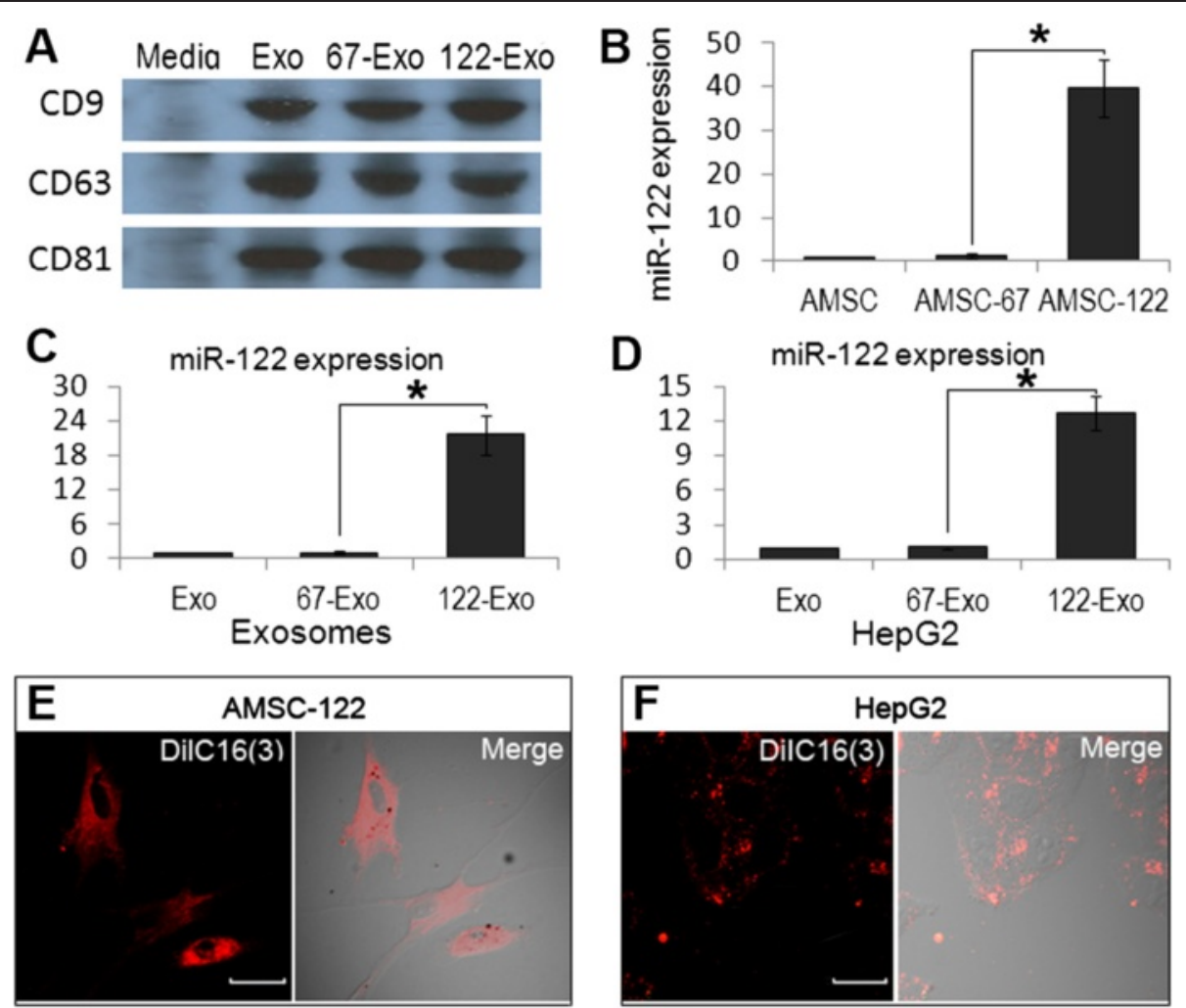

Fig. 2 Exosome-mediated miR-122 communication between AMSCs and HepG2 cells. a Western blot for CD9, CD63, and CD81 expression in AMSC-derived exosomes. b-d Real-time PCR detection of miR-122 expression in AMSCS (b), AMSC-derived exosomes (c), and exosome-treated HepG2 cells (d). e, $\mathbf{f}$ Confocal images of AMSC-122 stained with DilC 16 (3), a phospholipid membrane dye. Transfer of fluorescent exosomes from AMSC-122 is apparent in HepG2 cell membranes and cytoplasm. Data are presented as means \pm SE. $\left.{ }^{*} p<0.05, n=3\right)$. AMSC-122 miR-122-transfected AMSC, AMSC-67 cel-miR-67-transfected AMSC, Media precipitates from AMSC-conditioned media, Exo naïve AMSC-derived exosomes, 122-Exo AMSC-122-derived exosomes, 67-Exo AMSC-67-derived exosomes. Scale bar $=20 \mu \mathrm{m}$

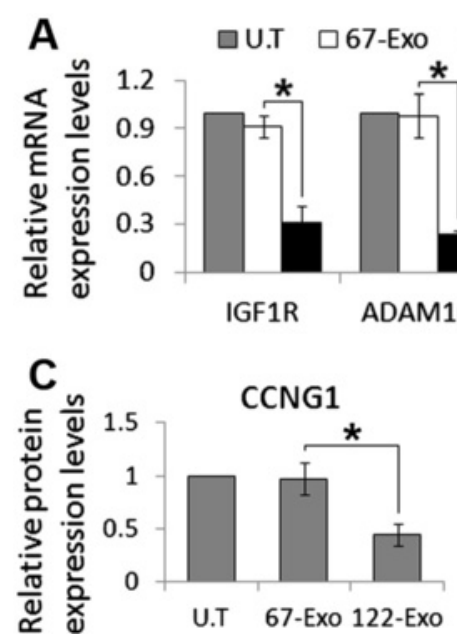

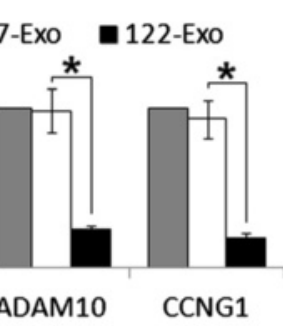

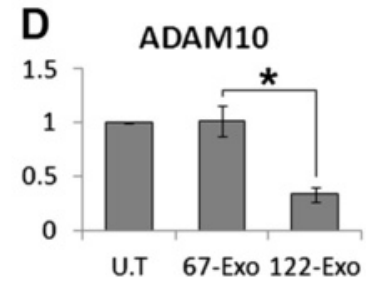

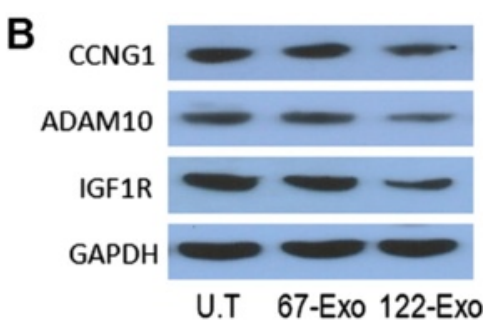

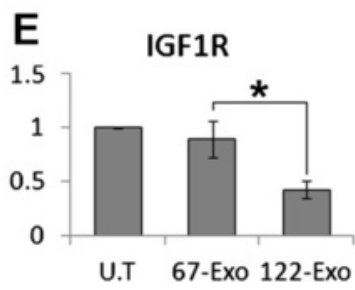

Fig. 3 122-Exo alters miR-122-target genes expression in HepG2 cells. a mRNA expression levels of miR-122-targeted genes in HepG2 cells treated with exosomes. b Western blot analysis of miR-122-targeted genes in HepG2 cells treated with exosomes. c-e Relative protein expression levels of CCNG1, ADAM10, and IGF1R in exosome-treated HepG2 cells. Data are presented as means \pm SE. $\left({ }^{*} p<0.05, n=3\right)$. U.T untreated, 122-ExO AMSC-122-derived exosomes, 67-Exo AMSC-67-derived exosomes 

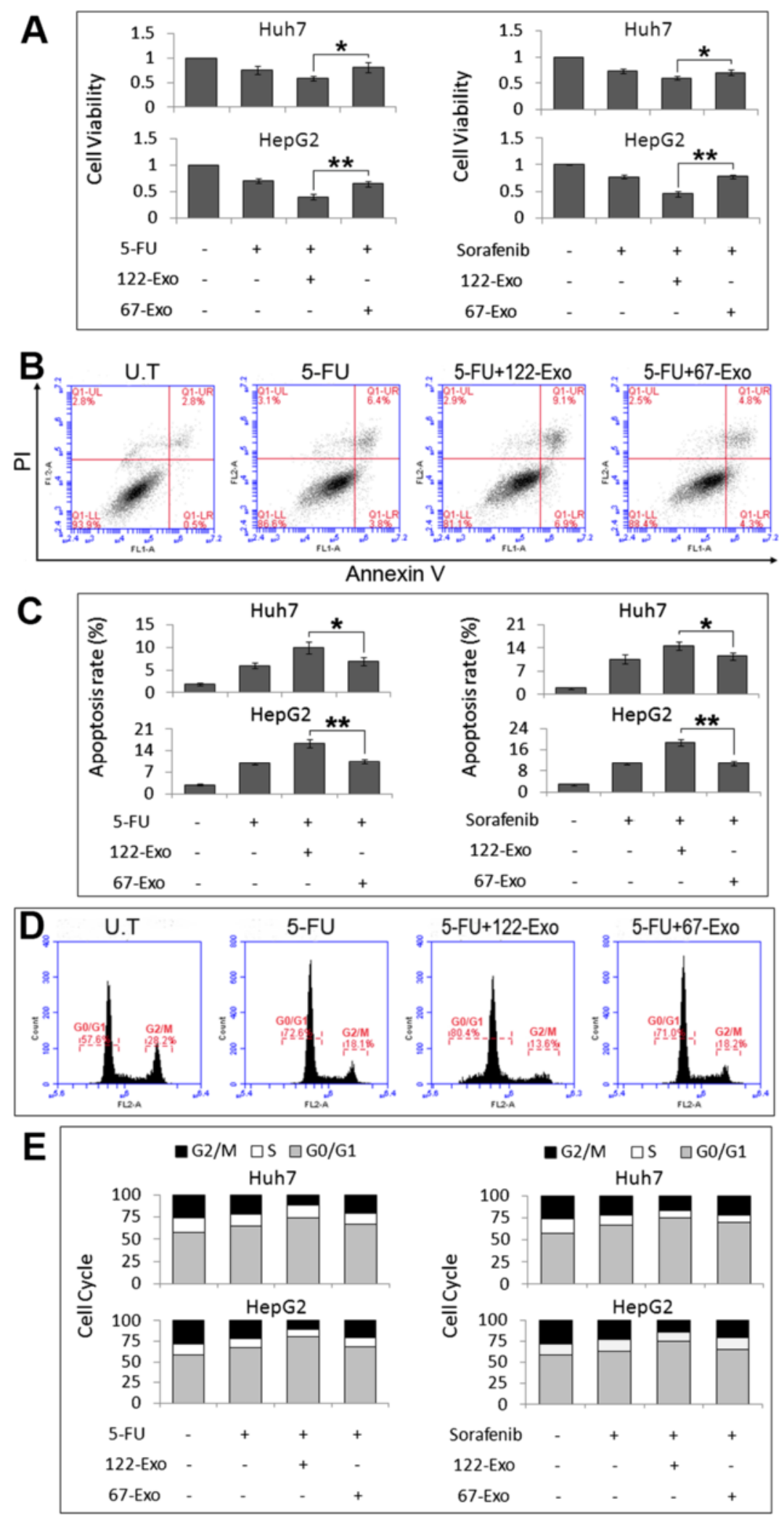

Fig. 4 (See legend on next page.) 
(See figure on previous page.)

Fig. 4 122-Exo sensitizes HCC cells to chemotherapeutic agents. a Cell viability assay on HepG2 and Huh7 cells by combined treatment with chemotherapeutic agents and AMSC-derived exosomes. b FITC-Annexin V/PI assay in exosome-treated HCC cells after 5-FU treatment. c FC analysis for Annexin $V$ revealed an increase in apoptotic cells in 122-Exo-treated HCC cells after 5-FU or sorafenib exposure. $\mathbf{d}$, e Cell cycle analysis revealed an increase in G0/G1 population in 122-ExO-treated HepG2 cells after 5-FU or sorafenib exposure. Data are presented as means \pm SE. $\left(^{*} p<0.05\right.$, ${ }^{* *} p<0.01$, $n=3)$. U.T untreated, 122-Exo AMSC-122-derived exosomes, 67-Exo AMSC-67-derived exosomes

and weight showed no difference between naïve exosomeor vehicle-treated mice at 28 days after exosome administration and sorafenib treatment (Fig. 5a, b). Real-time polymerase chain reaction (PCR) and Western blot analysis also showed that the expression of CCNG1, IGF1R, and ADAM10 gene was downregulated in 122-Exo-treated tumors in comparison with 67-Exo-treated cells (Fig. 5d, e). The expression of apoptosis-related genes, Caspase 3 and Bax (Bcl-2 Associated X protein), was upregulated in the 122-Exo-treated group. However, treatment with AMSCderived exosomes alone presented no effect on HCC growth. As shown in Fig. 6, no statistically significant difference was observed between exosome-treated groups and vehicle group in terms of the tumor volumes and weights at 28 days after exosome administration alone (without combination with sorafenib).
Overall, these results revealed that 122-Exo administration promotes the growth inhibitory property of sorafenib toward HCC cells.

\section{Discussion}

Exosomes are nanometer-sized vesicles of endocytic origin that are released by multiple cell types. Exosomes essentially exert their functions as mediators of intercellular communication by transferring protein and RNA. Thus, the use of exosomes as biological delivery vehicles is of considerable interest [21, 22]. Increasing studies indicate that MSCs are well suited for the mass production of exosomes [17], which may perform important functions in the therapeutic effect of MSCs through paracrine mechanism. MSCs have been reported to potentially regulate neurite outgrowth by exosome-mediated transfer of miR-

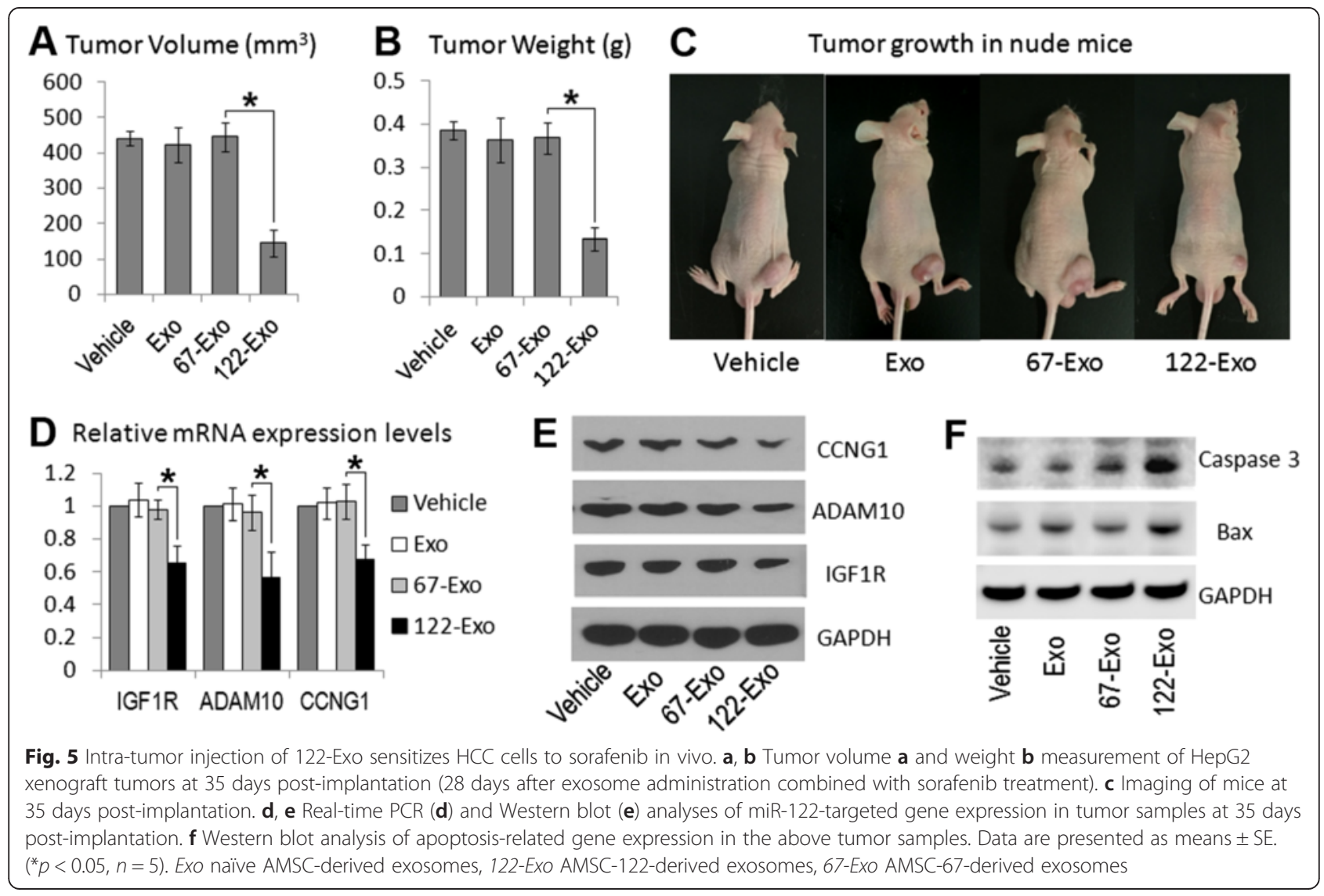



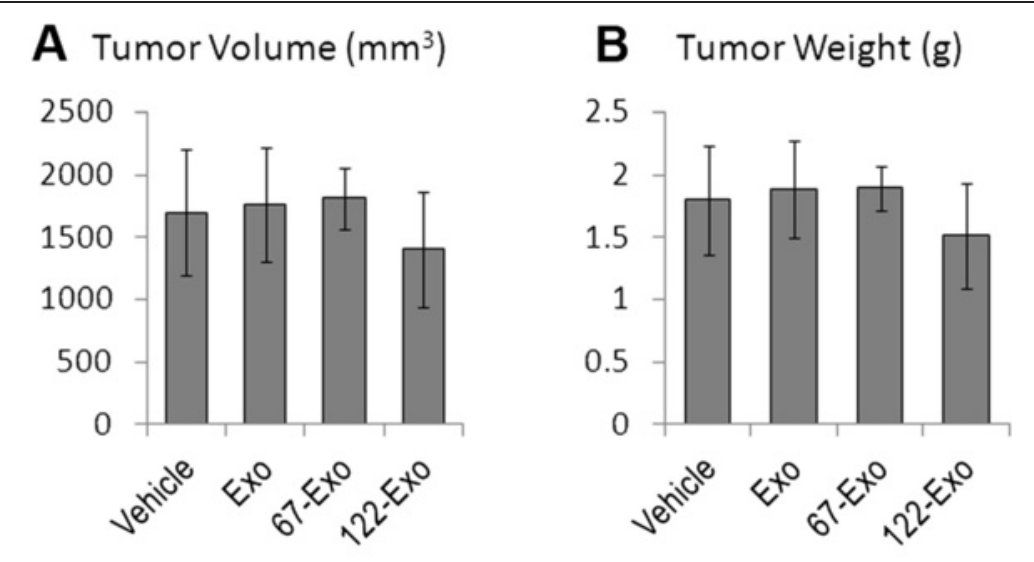

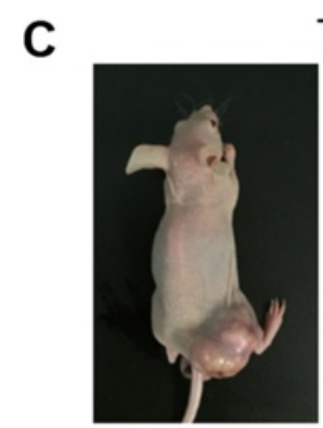

Vehicle

Tumor growth in nude mice

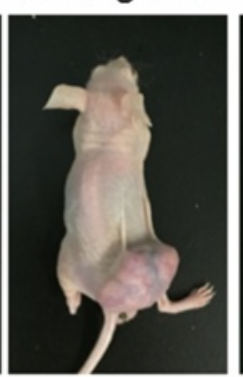

Exo

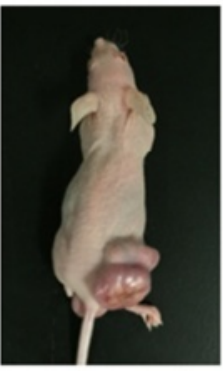

67-Exo

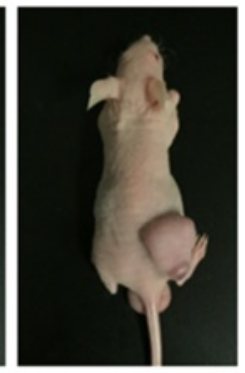

122-Exo

Fig. 6 Treatment with AMSC-derived exosomes alone has no effect on HCC growth in vivo. a, b Nude mice were inoculated subcutaneously with HepG2 cells. After 7 days of tumor growth, AMSC-derived exosomes were directly administered via intra-tumor injection. Tumor volume (a) and weight (b) measurement of HepG2 xenograft tumors at 35 days post-implantation (28 days after exosome administration). c Imaging of mice at 28 days after sole exosome treatment. Data are presented as means \pm SE $(n=5)$. Exo naïve AMSC-derived exosomes, 122-Exo AMSC-122-derived exosomes, 67-Exo AMSC-67-derived exosomes

$133 \mathrm{~b}$ to neural cells [23]. Further works show that exosomes derived from miR-146-expressing MSCs can deliver miR-146 into glioma cells in vitro, as well as reduce glioma xenograft growth in a rat model of primary brain tumor [16]. The present study demonstrated a novel strategy for increasing HCC chemosensitivity through AMSCs exosome-mediated transfer of therapeutic miR-122. The miR-122-modified AMSCs can effectively package miR122 into secreted exosomes, which mediate miR-122 communication between AMSCs and HCC cells, thus further increasing the sensitivity of HCC cells to chemotherapeutic agents through alteration of miR-122-target gene expression in these cells.

Among the predicted targets of miR-122, ADAM10, IGF1R, and CCNG1 play key roles in tumorigenesis and drug sensitivity in various cancers. ADAM10 is associated with tumor progression and confers resistance to doxorubicin-induced apoptosis in HCC cells by activation of the PI3-K/Akt pathway [24]. Signaling through IGF1R regulates HCC initiation, progression, metastasis, and resistance to therapy $[25,26]$. Enhanced expression of cyclin G1 (CCNG1) contributed to drug resistance of hepatoma cells and increased recurrence rate in HCC patients [12]. In vitro experiments indicated that 122Exo treatment resulted in the reduced expression of these genes in HCC cells over time, even at 4 weeks after122-Exo treatment (data not shown). In addition, an increase in the G0/G1-phase population and a corresponding decrease in the G2/M-phase, which is identified as a response to cyclin G1 knockdown [27], were observed in 122-Exo-treated HepG2 cells combined with chemotherapeutic agents. Therefore, by downregulating the expression levels of target genes, exosomes from miR-122-modified AMSCs can effectively increase the chemosensitivity of HepG2 cells through the induction of apoptosis and cell cycle arrest.

Given that systemic therapy with the multikinase inhibitor sorafenib is the standard of care for unresectable or advanced-stage HCC [28], we further tested whether 122-Exo can synergize its inhibitory function in mice model. A lower concentration of sorafenib $(5 \mathrm{mg} / \mathrm{kg})$ than the previously reported amount [29] was used to treat HepG2 xenograft tumors. One intra-tumor injection of 122-Exo significantly enhanced the growth 
inhibition by sorafenib at reduced concentration. However, the function of MSC in tumor therapy remains controversial [30]. Several studies have shown that MSCderived naïve MVs/exosomes can inhibit tumor growth in mice models of ovarian cancer, hepatoma, multiple myeloma, and bladder tumors [31-34]. Another study reported that MSC-derived exosomes can promote vascularity and tumor growth in mice xenograft models of gastric carcinoma and colon cancer [35]. We further tested whether naïve AMSC-derived exosomes can affect tumor growth. Naive exosomes were administered into nude mice bearing HepG2 cells through one intra-tumor injection, and PBS was used as vehicle control. No significant differences were observed between the two groups in terms of tumor volume and weight at 28 days after exosome administration combined with (Fig. 5) or without sorafenib treatment (Fig. 6). These data suggest that naïve MSC-derived exosomes may not affect HCC growth and chemosensitivity by themselves. The different effect of MSC-derived exosomes on tumor growth between our study and the above studies may be ascribed to differences in tumor-bearing models, MSCs sources, and route for exosome administration. Thus, the increased sensitivity of HCC cells to sorafenib by 122-Exo administration depends on exosome-mediated miR-122 transfer and downregulation of miR-122-target genes, which was reported to be involved in the antitumor activity of sorafenib in vivo $[36,37]$. However, treatment with 122-Exo alone (without combination with sorafenib) cannot significantly reduce tumor volume and weight (Fig. 6). This effect may be attributed to the possibility that without the growth retardation effect of sorafenib, one intra-tumor injection of 122-Exo was insufficient for tumor growth inhibition.

The current study directly delivered exosomes into the subcutaneous xenograft models via intra-tumor injection. A previous study demonstrated that by engineering the dendritic cells to express an exosomal membrane protein (Lamp2b) fused to $\alpha v$ integrin-specific iRGD peptide, natural exosomes can be used for targeted tumor therapy [38]. Additional work is needed to characterize the delivery of exosomal miRNAs in orthotopic HCC models via systemic administration. Moreover, considering that the isolation of exosomes and the culturing of MSCs include reagents and methods that are still inappropriate for human use, safety data from animal studies cannot ensure the safety of initial studies in humans. Improvement in the methods for AMSC culture and exosome purification will increase the feasibility and safety of AMSC-derived exosome therapy in clinical applications.

\section{Conclusions}

Our data indicate that miR-122, which is delivered via AMSC exosomes, can increase the sensitivity of $\mathrm{HCC}$ cells to chemotherapeutic agents, thereby providing a new treatment strategy for HCC.

\section{Methods}

\section{Isolation and identification of AMSCs}

Subcutaneous adipose tissues were obtained from three female patients (32, 28, and 41 years old, respectively) undergoing tumescent liposuction at the First Affiliated Hospital in Hangzhou. This study was approved by the hospital's ethics committee, and informed consent was obtained from each patients. Adipose tissue was processed as previously described [39] and maintained in Mesen$\mathrm{PRO}^{\circ} \mathrm{RS}^{\mathrm{Tm}}$ Medium (Gibco) containing $1 \%$ antibioticantimycotic (Gibco). The phenotype profile of AMSCs (passages 3 to 6) was evaluated through flow cytometry analysis (BD Accuri $\mathrm{C} 6$ flow cytometer) by using PElabeled cluster designation 29 (CD29), CD31, CD44, CD45, CD73, CD90, CD105, and human leukocyte antigen-DR (HLA-DR) (BD Bioscience Pharmingen) antibodies [39, 40]. IgG1 was used as isotype control. The differentiation of AMSCs to chondrocytes and adipocytes was tested by using StemPro ${ }^{\circ}$ chondrogenesis and adipogenesis differentiation kit (Gibco). Afterward, staining with Oil Red O and Alcian Blue was performed to detect adipocytes and chondrocytes, respectively [41].

\section{Cell culture}

HepG2 cells were maintained in DMEM (Gibco) containing $10 \%$ FBS (Gibco) and $1 \%$ antibiotic-antimycotic.

\section{Plasmids and AMSC transfection}

The AMSC-conditioned medium consisted of DMEM supplemented with $10 \%$ fetal bovine serum (FBS) from which bovine exosomes and protein aggregates were removed by ultracentrifugation at $100,000 \mathrm{~g}$ and $4{ }^{\circ} \mathrm{C}$ for $16 \mathrm{~h}$. Prior to transfection, $1 \times 10^{6}$ AMSCs were seeded in $10 \mathrm{~mL}$ of AMSC-conditioned medium overnight. AMSCs were then transfected with plasmids of hsamiR-122 or cel-miR-67, which contained no known mRNA-binding targets in human (GenScript) by using Lipofectamine $^{\text {tw }} 2000$ (Invitrogen). At $48 \mathrm{~h}$ after transfection, AMSCs were harvested for real-time PCR analysis.

\section{Isolation and identification of AMSC-derived exosomes}

After $48 \mathrm{~h}$ of miRNA transfection, exosomes were isolated from the AMSCs supernatant by using an ExoQuick-TC Kit (System Biosciences, CA) in accordance with the manufacturer's instructions. These exosomes were then characterized by Western blotting analysis of exosome surface markers, such as CD9, CD63, and CD81 (Abcam). Precipitates from AMSC-conditioned media were used as negative control. The protein content of exosomes was determined by using $\mathrm{BCA}$ protein assay kit (Pierce). 
Subsequently, exosome pellets were resuspended in sterile PBS at a total protein concentration of $5 \mu \mathrm{g} / \mu \mathrm{L}$.

\section{Isolation and detection of miRNA}

Total RNA enriched with miRNAs was isolated from AMSCs or exosomes by using a miRVana miRNA isolation kit. Real-time PCR was then performed following the manufacturer's instructions (Ambion Diagnostics, TX) to examine miR-122 expression. Data were normalized over the average cycle threshold (CT) value of U6, and $2^{-\Delta \Delta C T}$ method was used to determine the relative miRNA expression.

\section{Confocal microscopy studies}

AMSCs were labeled with the phospholipid membrane dye, lipophilic carbocyanine $\mathrm{DilC}_{16}$ (3) $(1.25 \mu \mathrm{M})$ [42]. After $10 \mathrm{~min}$ of incubation at $37^{\circ} \mathrm{C}$, cells were washed and resuspended in fresh media for $48 \mathrm{~h}$. Fluorescent exosomes were collected and added into recipient HepG2 cells. Afterward, cells were fixed with methanol, mounted on slides, and imaged via confocal microscopy (Olympus). Background fluorescence was subtracted using unstained cells.

\section{RNA isolation and real-time PCR}

Total RNA was isolated from HepG2 cells or tumor samples by using TRIzol, followed by real-time PCR analysis with ABI Prism 7900 (Applied Biosystems, Foster City, CA) to examine the expression of CCNG1, ADAM10, and IGF1R. GAPDH was used as an internal control. The $2^{-\triangle \Delta C T}$ method was employed to determine the relative mRNA expression.

\section{Western blot analysis}

HepG2 cells or tumor samples were lysed with RIPA peptide lysis buffer (Beyotime Biotechnology, Jiangsu, China) containing $1 \%$ protease inhibitors (Pierce). The protein content of different fractions was detected via BCA method. Equivalent amounts of protein $(20 \mu \mathrm{g})$ were separated by $10 \%$ SDS-PAGE gels and transferred to polyvinylidene difluoride membranes (Millipore, Bedford, MA) and blocked with $1 \%$ BSA in TBST for $1 \mathrm{~h}$ at room temperature. The membrane was incubated with CCNG1, IGF1R, ADAM10, Bax, Caspase 3, or GAPDH (Abcam) antibodies overnight at $4{ }^{\circ} \mathrm{C}$. After washing, the membrane was incubated with HRP-conjugated secondary antibody (1:3000; Huabio) for $1 \mathrm{~h}$. Blots were visualized via ECL-associated fluorography (Millipore).

\section{Cell viability assay}

HepG2 and Huh7 cells were plated in 96-well plates at the concentration of $2 \times 10^{3} /$ well and treated with 5-FU or sorafenib combined with or without exosomes (50 ng/ $/ \mathrm{L}$ ). At $72 \mathrm{~h}$ after culture, cell viability was determined by an 3-(4,5-dimethyl-2-thiazolyl)-2,5-diphenyl-2-H-tetrazolium bromide (MTT) assay (Sigma).

\section{Flow cytometry analysis of cell apoptosis and cell cycle}

Cells were plated in 6-well plates at the concentration of $2 \times 10^{5} /$ well and treated with 5 -FU $(10 \mu \mathrm{M})$ or sorafenib $(5 \mu \mathrm{M})$ combined with or without exosomes $(50 \mathrm{ng} / \mu \mathrm{L})$. At $48 \mathrm{~h}$ after treatment, cell apoptosis and cell cycle were detected using an Annexin V/PI detection kit (BD Biosciences) and cell-cycle staining kit (MultiSciences), in accordance with the manufacturer's instructions and then analyzed on a BD Accuri ${ }^{\ominus}$ C6 flow cytometer.

\section{Xenograft models and exosome treatment}

Male Balb/c nude mice (6 weeks old) were purchased from Zhejiang Academy of Medical Science. All experimental procedures were conducted in accordance with the Chinese legislation regarding experimental animals. Mice were inoculated subcutaneously with HepG2 cells $\left(5 \times 10^{6}\right)$. After 7 days of tumor growth, mice were randomized into eight groups prior to exosome treatment. The groups comprised four groups for sole exosome administration and another four groups for exosome administration combined with sorafenib treatment. Exo (naive MSC-derived exosomes), 67-Exo (AMSC-67-derived exosomes), or 122-Exo (AMSC-122-derived exosomes) suspension (50 $\mu \mathrm{g}$ total protein in $10 \mu \mathrm{L}$ volume) was directly administered via intra-tumor injection into each mice ( $n=5$ /group), respectively. PBS was used as vehicle control. Sorafenib $(5 \mathrm{mg} / \mathrm{kg}$ ) was administered by intraperitoneal (i.p.) injection for five consecutive days of each week. Tumor volumes were calculated using the following formula: tumor volume $\left(\mathrm{mm}^{3}\right)=$ $0.5 \times(W)^{2} \times(L)$, where $L$ represents the length and $W$ represents the width.

\section{Statistical analysis}

Differences between groups were analyzed by using conventional Student's $t$ test or ANOVA. Each experiment was repeated at least thrice, and data were presented as mean \pm SE (standard error). A $p$ value of 0.05 or less was considered as statistically significant.

\section{Abbreviations}

122-Exo: AMSC-122-derived exosomes; 67-Exo: AMSC-67-derived exosomes; ADAM10: A distintegrin and metalloprotease 10; AMSC-122: miR-122transfected AMSCs; AMSC-67: Cel-miR-67-transfected AMSCs; AMSCs: Adipose tissue-derived MSCs; CCNG1: Cyclin G1; Exo: Naïve AMSC-derived exosomes; HCC: Hepatocellular carcinoma; i.p.: Intra-peritoneal; IGF1R: Insulin-like growth factor receptor 1; MSCs: Mesenchymal stem cells; MVs: Microvesicles; TACE: Transarterial chemoembolization; U.T: Untreated.

\section{Competing interests}

The authors declare that they have no competing interests. 


\section{Authors' contributions}

GL performed the experimental work and drafted the manuscript. XS, FY, SW, and JW participated in the experiments and performed the statistical analysis. ZC and YL conceived of the study and participated in its design and coordination. All authors read and approved the final manuscript.

\section{Acknowledgements}

The work was supported by the National Natural Science Fund $(81000730$ and 81201782), the International Science and Technology Cooperation Project (re-innovation industrialization) (2012C14028), and the Medical and Health science and technology project in Zhejiang province (2014KYB085).

\section{Author details}

${ }^{1}$ State Key Laboratory for Diagnosis and Treatment of Infectious Diseases, The First Affiliated Hospital, College of Medicine, Zhejiang University, Collaborative Innovation Center for Diagnosis and Treatment of Infectious Diseases, 79\# Qingchun Road, 6A-17, Hangzhou 310003, China. ${ }^{2}$ Institute of Genetics, College of Life Science, Zhejiang University, Hangzhou 310003, China.

Received: 26 August 2015 Accepted: 13 October 2015 Published online: 29 October 2015

\section{References}

1. Bruix J, Sherman M. American Association for the Study of Liver Diseases. Management of hepatocellular carcinoma. Hepatology. 2005;42:1208-36.

2. Zhang KZ, Zhang QB, Zhang QB, Sun HC, Ao JY, Chai ZT, et al. Arsenic trioxide induces differentiation of CD133+ hepatocellular carcinoma cells and prolongs posthepatectomy survival by targeting GLI1 expression in a mouse model. J Hematol Oncol. 2014;7:28.

3. Smith AD, Roda D, Yap TA. Strategies for modern biomarker and drug development in oncology. J Hematol Oncol. 2014;7(1):70.

4. Zhang $H$, Chen Z, Wang X, Huang Z, He Z, Chen Y. Long non-coding RNA: a new player in cancer. J Hematol Oncol. 2013;6:37.

5. Wang WT, Chen YQ. Circulating miRNAs in cancer: from detection to therapy. J Hematol Oncol. 2014;7(1):86.

6. Braoudaki M, Lambrou Gl, Giannikou K, Milionis V, Stefanaki K, Birks DK, et al. Microrna expression signatures predict patient progression and disease outcome in pediatric embryonal central nervous system neoplasms. J Hematol Oncol. 2014;7(1):96.

7. Fan $S$, Chen $W X$, Lv XB, Tang QL, Sun LJ, Liu BD, et al. miR-483-5p determines mitochondrial fission and cisplatin sensitivity in tongue squamous cell carcinoma by targeting FIS1. Cancer Lett. 2015;362:183-91.

8. Sarkar FH, Li Y, Wang Z, Kong D, Ali S. Implication of microRNAs in drug resistance for designing novel cancer therapy. Drug Resist Update. 2010;13(3):57-66.

9. Bandiera S, Pfeffer S, Baumert TF, Zeisel MB. miR-122-a key factor and therapeutic target in liver disease. J Hepatol. 2015;62:448-57.

10. Tsai WC, Hsu SD, Hsu CS, Lai TC, Chen SJ, Shen R, et al. MicroRNA-122 plays a critical role in liver homeostasis and hepatocarcinogenesis. J Clin Invest. 2012;122:2884-97.

11. Coulouarn C, Factor VM, Andersen JB, Durkin ME, Thorgeirsson SS. Loss of miR-122 expression in liver cancer correlates with suppression of the hepatic phenotype and gain of metastatic properties. Oncogene. 2009;28:3526-36

12. Xu Y, Xia F, Ma L, Shan J, Shen J, Yang Z, et al. MicroRNA-122 sensitizes HCC cancer cells to adriamycin and vincristine through modulating expression of MDR and inducing cell cycle arrest. Cancer Lett. 2011;310(2):160-9.

13. Fornari F, Gramantieri L, Giovannini C, Veronese A, Ferracin M, Sabbioni S, et al. MiR-122/cyclin G1 interaction modulates p53 activity and affects doxorubicin sensitivity of human hepatocarcinoma cells. Cancer Res. 2009;69:5761-7.

14. Bai S, Nasser MW, Wang B, Hsu SH, Datta J, Kutay H, et al. MicroRNA-122 inhibits tumorigenic properties of hepatocellular carcinoma cells and sensitizes these cells to sorafenib. J Biol Chem. 2009:284:32015-27.

15. $\mathrm{Hu} \mathrm{G}$, Drescher KM, Chen XM. Exosomal miRNAs: biological properties and therapeutic potential. Front Genet. 2012;3:56.

16. Katakowski M, Buller B, Zheng X, Lu Y, Rogers T, Osobamiro O, et al. Exosomes from marrow stromal cells expressing miR-146b inhibit glioma growth. Cancer Lett. 2013;335:201-4.
17. Yeo RW, Lai RC, Zhang B, Tan SS, Yin Y, Teh BJ, et al. Mesenchymal stem cell: an efficient mass producer of exosomes for drug delivery. Adv Drug Deliv Rev. 2013;65:336-41.

18. Lai RC, Arslan F, Lee MM, Sze NS, Choo A, Chen TS, et al. Exosome secreted by MSC reduces myocardial ischemia/reperfusion injury. Stem Cell Res. 2010;4:214-22.

19. Schäffler A, Büchler C. Concise review: adipose tissue-derived stromal cells-basic and clinical implications for novel cell-based therapies. Stem Cells. 2007;25:818-27.

20. Kumar D, Gupta D, Shankar S, Srivastava RK. Biomolecular characterization of exosomes released from cancer stem cells: possible implications for biomarker and treatment of cancer. Oncotarget. 2015;6(5):3280-91.

21. Zhang X, Yuan X, Shi H, Wu L, Qian H, Xu W. Exosomes in cancer: small particle, big player. J Hematol Oncol. 2015;8:83.

22. O'Loughlin AJ, Woffindale CA, Wood MJ. Exosomes and the emerging field of exosome-based gene therapy. Curr Gene Ther. 2012;12:262-74.

23. Xin H, Li Y, Buller B, Katakowski M, Zhang Y, Wang X, et al. Exosomemediated transfer of miR-133b from multipotent mesenchymal stromal cells to neural cells contributes to neurite outgrowth. Stem Cells. 2012;30:1556-64.

24. Yang $C$, Jiang F, Xu F, Jiang G. ADAM10 overexpression confers resistance to doxorubicin-induced apoptosis in hepatocellular carcinoma. Tumour Biol. 2012;33:1535-41

25. Wu J, Zhu AX. Targeting insulin-like growth factor axis in hepatocellular carcinoma. J Hematol Oncol. 2011;5:30.

26. Tovar V, Alsinet C, Villanueva A, Hoshida Y, Chiang DY, Solé M, et al. IGF activation in a molecular subclass of hepatocellular carcinoma and preclinical efficacy of IGF-1R blockage. J Hepatol. 2010;52:550-9.

27. Kimura SH, Ikawa M, Ito A, Okabe M, Nojima H. Cyclin G1 is involved in G2/M arrest in response to DNA damage and in growth control after damage recovery. Oncogene. 2001;20:3290-300.

28. Wilhelm SM, Adnane L, Newell P, Villanueva A, Llovet JM, Lynch M. Preclinical overview of sorafenib, a multikinase inhibitor that targets both Raf and VEGF and PDGF receptor tyrosine kinase signaling. Mol Cancer Ther. 2008;7:3129-40.

29. Liang $Y$, Zheng $T$, Song $R$, Wang J, Yin D, Wang L, et al. Hypoxia-mediated sorafenib resistance can be overcome by EF24 through Von Hippel-Lindau tumor suppressor-dependent HIF-1a inhibition in hepatocellular carcinoma. Hepatology. 2013;57:1847-57.

30. Sun Z, Wang S, Zhao RC. The roles of mesenchymal stem cells in tumor inflammatory microenvironment. J Hematol Oncol. 2014;7:14.

31. Roccaro AM, Sacco A, Maiso P, Azab AK, Tai YT, Reagan M, et al. BM mesenchymal stromal cell-derived exosomes facilitate multiple myeloma progression. J Clin Invest. 2013;123:1542-55.

32. Bruno S, Collino F, Deregibus MC, Grange C, Tetta C, Camussi G. Microvesicles derived from human bone marrow mesenchymal stem cells inhibit tumor growth. Stem Cells Dev. 2013;22:758-71.

33. Wu S, Ju G, Du T, et al. Microvesicles derived from human umbilical cord Wharton's jelly mesenchymal stem cells attenuate bladder tumor cell growth in vitro and in vivo. PloS One. 2013;8, e61366.

34. Akyurekli C, Le Y, Richardson RB, Fergusson D, Tay J, Allan DS. A systematic review of preclinical studies on the therapeutic potential of mesenchymal stromal cell-derived microvesicles. Stem Cell Rev. 2015;11:150-60.

35. Zhu W, Huang L, Li Y, Zhang X, Gu J, Yan Y, et al. Exosomes derived from human bone marrow mesenchymal stem cells promote tumor growth in vivo. Cancer Lett. 2012;315:28-37.

36. Zhang W, Liu S, Liu K, Ji B, Wang Y, Liu Y. Knockout of ADAM10 enhances sorafenib antitumor activity of hepatocellular carcinoma in vitro and in vivo. Oncol Rep. 2014;32(5):1913-22

37. Ou DL, Lee BS, Chang YC, Lin LI, Liou JY, Hsu C, et al. Potentiating the efficacy of molecular targeted therapy for hepatocellular carcinoma by inhibiting the insulin-like growth factor pathway. PLoS One. 2013;8, e66589.

38. Tian Y, Li S, Song J, Ji T, Zhu M, Anderson GJ, et al. A doxorubicin delivery platform using engineered natural membrane vesicle exosomes for targeted tumor therapy. Biomaterials. 2014;35:2383-90.

39. Liu Y, Yan X, Sun Z, Chen B, Han Q, Li J, et al. Flk- $1^{+}$adipose-derived mesenchymal stem cells differentiate into skeletal muscle satellite cells and ameliorate muscular dystrophy in mdx mice. Stem Cells Dev. 2007;16:695-706.

40. Dudakovic A, Camilleri E, Riester SM, Lewallen EA, Kvasha S, Chen X, et al. High-resolution molecular validation of self-renewal and spontaneous differentiation in clinical-grade adipose-tissue derived human mesenchymal stem cells. J Cell Biochem. 2014;115(10):1816-28. 
41. Ullah M, Stich S, Notter M, Eucker J, Sittinger M, Ringe J. Transdifferentiation of mesenchymal stem cells-derived adipogenic-differentiated cells into osteogenic- or chondrogenic-differentiated cells proceeds via dedifferentiation and have a correlation with cell cycle arresting and driving genes. Differentiation. 2013;85(3):78-90.

42. Ismail N, Wang Y, Dakhlallah D, Moldovan L, Agarwal K, Batte K, et al. Macrophage microvesicles induce macrophage differentiation and miR-223 transfer. Blood. 2013;121(6):984-95.

Submit your next manuscript to BioMed Central and take full advantage of:

- Convenient online submission

- Thorough peer review

- No space constraints or color figure charges

- Immediate publication on acceptance

- Inclusion in PubMed, CAS, Scopus and Google Scholar

- Research which is freely available for redistribution 